PROCEEDINGS OF THE

AMERICAN MATHEMATICAL SOCIETY

Volume 132, Number 7 , Pages 1885-1890

S 0002-9939(04)07383-6

Article electronically published on January 7, 2004

\title{
A CHARACTERIZATION OF SEMI-QUASIHOMOGENEOUS FUNCTIONS IN TERMS OF THE MILNOR NUMBER
}

\author{
MASAKO FURUYA AND MASATAKA TOMARI \\ (Communicated by Michael Stillman)
}

\begin{abstract}
We give an inequality on the Milnor number $\mu(f)$ of a hypersurface isolated singularity in terms of the weighted Taylor expansion $f=f_{\rho}+f_{\rho+1}+$ $\cdots$ for a given weight on the coordinates. Here the equality holds if and only if the leading term $f_{\rho}$ defines an isolated singularity. This gives a characterization of the semi-quasihomogeneous condition in terms of $\mu$. Our proof uses a result on multiplicity of filtered rings and is given by purely algebraic arguments.
\end{abstract}

\section{INTRODUCTION}

Let $f \in \mathbf{C}\left\{x_{1}, \ldots, x_{d}\right\}$ define an isolated singularity at the origin. The Milnor number $\mu(f)$ is defined and computed by the relation (see [G])

$$
\mu(f)=\operatorname{dim}_{\mathbf{C}} \mathbf{C}\left\{x_{1}, \ldots, x_{d}\right\} /\left(\frac{\partial f}{\partial x_{1}}, \ldots, \frac{\partial f}{\partial x_{d}}\right) .
$$

Let $w=\left(w_{1}, \ldots, w_{d}\right)$ be a weight on the coordinates $\left(x_{1}, \ldots, x_{d}\right)$ by positive integers $w_{i}, i=1, \ldots, d$. We have the weighted Taylor expansion $f=f_{\rho}+f_{\rho+1}+\cdots$ with respect to $w$ and $f_{\rho} \neq 0$, where $f_{k}$ is a quasihomogeneous polynomial of type $\left(w_{1}, \ldots, w_{d+1} ; k\right)$, for $k \geq \rho$. Then our main theorem is stated as follows:

Theorem 1. Let the situation be as above. Then:

(1) The following inequality holds:

$$
\mu(f) \geq\left(\frac{\rho}{w_{1}}-1\right) \cdots\left(\frac{\rho}{w_{d}}-1\right) .
$$

(2) The equality holds in the above if and only if $f_{\rho}$ defines an isolated singularity at the origin.

Here we recall that $f$ is called a semi-quasihomogeneous function if the initial term $f_{\rho}$ defines an isolated singularity at the origin. It is well known that Lê Dũng Tráng and Ramanujam [4] and Arnol'd [1] showed independently that the equality in (1) holds for a semi-quasihomogeneous function. Furthermore, their formula is a natural extension of Milnor and Orlik's formula [7] for a quasihomogeneous

Received by the editors June 28, 2001 and, in revised form, March 15, 2003.

2000 Mathematics Subject Classification. Primary 14B05; Secondary 13H15, 32S05.

Key words and phrases. Milnor number, semi-quasihomogeneous function, multiplicity of local rings, filtered rings. 
function. Now our Theorem 1 generalizes their formula and characterizes the semiquasihomogeneous condition of $f$ by means of the equivalence condition of (2).

In the above theorem, (1) was first proved by Furuya [2] by using Kouchinirenko's result [3], where Furuya strengthened (1) in the following form:

$$
\mu(f)=\mu(\tilde{f}) \geq \nu\left(\Gamma_{-}(\tilde{f})\right) \geq\left(\frac{\rho}{w_{1}}-1\right) \cdots\left(\frac{\rho}{w_{d}}-1\right)
$$

where $\tilde{f}$ is a standard modification of $f$ to a convenient function and $\nu\left(\Gamma_{-}(\tilde{f})\right)$ is the Newton number in Kouchinirenko's sense; cf. [2] and 3] for general facts about the Newton boundary of $f$. The proof of this note is different from that of [2], and uses a theory of multiplicity of filtered rings. By this idea, we also prove the necessary and sufficient condition where the equality holds. Though our argument gives no information about the Newton number $\nu\left(\Gamma_{-}(\tilde{f})\right)$, it is very short. So we decided to publish this other proof in this note.

See [6] and [7] for the basic facts on the Milnor number, and [5] for those on the multiplicity of local rings.

\section{$\S 1$. A REVIEW OF A THEOREM ON MULTiPliCity OF FILTERED RINGS}

Let $(A, m)$ be a $d$-dimensional local ring that is defined as the local ring of some scheme over an infinite field and analytically unramified. A filtration $F=\left\{F^{k}\right\}_{k \geq 0}$ is a decreasing sequence of ideals of $A$ that satisfies $F^{0}=A, F^{1}=m, F^{k} \supset F^{k+1}$, and $F^{k} \cdot F^{j} \subset F^{k+j}$. We always assume that $\mathcal{R}=\bigoplus_{k>0} F^{k} \cdot T^{k} \subset A[T]$ is a finitely generated $A$-algebra. Here $T$ is an indeterminate. There is a positive integer $N$ such that $F^{k N}=\left(F^{N}\right)^{k}$ holds for $k \geq 0$, and we assume that $F^{N}$ is $m$-primary. One can find general information on the theory of filtered rings and filtered blowing-up in 10. Let $y_{1}, \ldots, y_{d} \in m$ be a system of parameters of $A$, and assume that the relation $y_{i} \in F^{a_{i}}$ holds for each $i$, where $a_{i} \in \mathbf{Z}_{\geq 1}$. Let $G=\bigoplus_{k>0} F^{k} / F^{k+1}$ be the associated graded ring, and let $P(G, \lambda)=\sum l\left(G_{k}\right) \lambda^{k} \in \mathbf{Z}[[\lambda]]$. Then the arguments of the proof of Theorem A of [9] yield the following result on the multiplicity $e\left(\left(y_{1}, \ldots, y_{d}\right), A\right)$.

Theorem 2. (1) We have the inequality

$$
e\left(\left(y_{1}, \ldots, y_{d}\right), A\right) \geq\left(\prod_{i=1}^{d} a_{i}\right) \lim _{\lambda \rightarrow 1}(1-\lambda)^{d} P(G, \lambda) .
$$

(2) The equality holds in the above if and only if the following two conditions hold: (2)-1. $y_{i} \in F^{a_{i}}-F^{a_{i}+1}$, for $i=1, \ldots, d$, and (2)-2. $\bar{y}_{i} \in G_{a_{i}}$, for $i=1, \ldots, d$, defines a system of parameters of $G$.

In the case of (2), we obtain the equality $e\left(\left(\overline{y_{1}}, \ldots, \overline{y_{d}}\right), G\right)=e\left(\left(y_{1}, \ldots, y_{d}\right), A\right)$.

A sketch of the proof. The arguments for " $y_{1}, \ldots, y_{d}$ " in the paragraphs (1.6) and (1.7) of 9] work for almost all of these claims. However, for the convenience of the readers, we give a sketch of the proof.

(1) Let $L$ be a positive integer divisible by L.C.M. $\left(a_{1}, \ldots, a_{d}\right)$. We have the relation $\left(y_{1}^{\frac{L}{a_{1}}}, \ldots, y_{d}^{\frac{L}{a_{d}}}\right) \subset F^{L}$ and the inequality $e\left(F^{L}, A\right) \leq e\left(\left(y_{1}^{\frac{L}{a_{1}}}, \ldots, y_{d}^{\frac{L}{a_{d}}}\right), A\right)$. We can choose $L$ so big that the relation $F^{k L}=F^{L} \ldots F^{L}$ (k times) holds for $k \geq 0$. Then we can show the relation (Lemma (1.3) of [9]) $e\left(F^{L}, A\right)=L^{d} \cdot \lim _{\lambda \rightarrow 1}(1-$ 
$\lambda)^{d} P(G, \lambda)$. By Lech's Lemma (Theorem (14.12) of [5]) we can show the equality

$$
e\left(\left(y_{1}^{\frac{L}{a_{1}}}, \ldots, y_{d}^{\frac{L}{a_{d}}}\right), A\right)=\frac{L}{a_{1}} \cdot \cdots \cdot \frac{L}{a_{d}} \cdot e\left(\left(y_{1}, \ldots, y_{d}\right), A\right) .
$$

Therefore we obtain the desired inequality.

(2) First we assume that the equality holds. Then the inequality (1) implies the condition (2)-1 obviously. We obtain the relation $e\left(F^{L}, A\right)=e\left(\left(y_{1}^{\frac{L}{a_{1}}}, \ldots, y_{d}^{\frac{L}{a_{d}}}\right), A\right)$. A theorem of D. Rees $[8]$ implies that $\left(y_{1}^{\frac{L}{a_{1}}}, \ldots, y_{d}^{\frac{L}{a_{d}}}\right)$ is a reduction of $F^{L}$. Then one can show that the initial forms $\bar{y}_{1}^{\frac{L}{a_{1}}}, \ldots, \bar{y}_{d}^{\frac{L}{a_{d}}}$ give a parameter system for $G$. In (1.7) of [9], Tomari proved this assertion by using the filtered blowing-up by $F$. Next we assume that the conditions (2)-1 and (2)-2 hold. We can show the relation (Lemma (1.5) of [9]) $e\left(\left(\bar{y}_{1}, \ldots, \bar{y}_{d}\right), G\right)=\left(\prod_{i=1}^{d} a_{i}\right) \lim _{\lambda \rightarrow 1}(1-\lambda)^{d} P(G, \lambda)$. Since $\operatorname{gr}_{F}\left(\left(y_{1}, \ldots, y_{d}\right)^{l}\right) \supset\left(\bar{y}_{1}, \ldots, \bar{y}_{d}\right)^{l}$ holds for $l \geq 0$, we can easily show the relation $e\left(\left(y_{1}, \ldots, y_{d}\right), A\right) \leq e\left(\left(\bar{y}_{1}, \ldots, \bar{y}_{d}\right), G\right)$. Hence, by the inequality (1), these are equal.

\section{§2. A PROOF OF THEOREM 1}

(1) Let $F=\left\{F^{k}\right\}$ be the filtration on $\mathbf{C}\left\{x_{1}, \ldots, x_{d}\right\}$ by the weight $w$ on the coordinates $x_{1}, \ldots, x_{d}$. We have the relation $f \in F^{\rho}-F^{\rho+1}$. Since $f$ defines an isolated singularity at the origin, $\frac{\partial f}{\partial x_{1}}, \ldots, \frac{\partial f}{\partial x_{d}}$ define a system of parameters of $\mathbf{C}\left\{x_{1}, \ldots, x_{d}\right\}$.

We have the weighted Taylor expansion $f=\sum_{k \geq \rho} f_{k}$ where $f_{k}$ is a weighted homogeneous polynomial of the type $\left(w_{1}, \ldots, w_{d} ; k\right)$. Furthermore, we have $\frac{\partial f}{\partial x_{i}}=$ $\sum_{k \geq \rho} \frac{\partial f_{k}}{\partial x_{i}}$. Here $\frac{\partial f_{k}}{\partial x_{i}}$ is zero or a weighted homogeneous polynomial of type $\left(w_{1}, \ldots, w_{d} ; k-w_{i}\right)$. Hence $\frac{\partial f}{\partial x_{i}} \in F^{\rho-w_{i}}$ for $i=1, \ldots, d$. By (1) of Theorem 2 , we obtain the relation

$$
e\left(\left(\frac{\partial f}{\partial x_{1}}, \ldots, \frac{\partial f}{\partial x_{d}}\right), \mathbf{C}\left\{x_{1}, \ldots, x_{d}\right\}\right) \geq\left(\prod_{i=1}^{d}\left(\rho-w_{i}\right)\right) \lim _{\lambda \rightarrow 1}(1-\lambda)^{d} P(G, \lambda) .
$$

Since $\mathbf{C}\left\{x_{1}, \ldots, x_{d}\right\}$ is Cohen-Macaulay, it is well known that

$$
e\left(\left(\frac{\partial f}{\partial x_{1}}, \ldots, \frac{\partial f}{\partial x_{d}}\right), \mathbf{C}\left\{x_{1}, \ldots, x_{d}\right\}\right)=\operatorname{dim} \mathbf{C}\left\{x_{1}, \ldots, x_{d}\right\} /\left(\frac{\partial f}{\partial x_{1}}, \ldots, \frac{\partial f}{\partial x_{d}}\right) .
$$

This coincides with $\mu(f)$.

By our definition of the filtration $F \cdot, G=\mathbf{C}\left[x_{1}, \ldots, x_{d}\right]$ and we can easily see the relation $P(G, \lambda)=\frac{1}{\left(1-\lambda^{w_{1}}\right) \cdots\left(1-\lambda^{w_{d}}\right)}$. Hence we obtain the relation $\lim _{\lambda \rightarrow 1}(1-\lambda)^{d} P(G, \lambda)=\frac{1}{w_{1} \cdots w_{d}}$. This completes the proof of $(1)$ of Theorem 1.

(2) Let us employ the notation of (1). First, we show the necessity part of our assertion. Suppose the equality holds in (1) of Theorem 1. Then by (2) of Theorem 2, we obtain the relations $\frac{\partial f}{\partial x_{i}} \in F^{\rho-w_{i}}-F^{\rho-w_{i}+1}$, for $i=1, \ldots, d$. 
Hence $\frac{\partial f_{\rho}}{\partial x_{i}} \neq 0$, and we may write $\frac{\overline{\partial f}}{\partial x_{i}} \in G$ as $\frac{\partial f_{\rho}}{\partial x_{i}} \in \mathbf{C}\left[x_{1}, \ldots, x_{d}\right]=G$. By Theorem $2, \frac{\partial f_{\rho}}{\partial x_{1}}, \ldots, \frac{\partial f_{\rho}}{\partial x_{d}}$ define a system of parameters at the origin. Hence $f_{\rho}$ defines an isolated singularity at the origin.

Next we show the sufficiency part. Assume that $f_{\rho}$ defines an isolated singularity. Then $\frac{\partial f_{\rho}}{\partial x_{i}} \neq 0$ for $i=1, \ldots, d$, and $\frac{\partial f_{\rho}}{\partial x_{1}}, \ldots, \frac{\partial f_{\rho}}{\partial x_{d}}$ define a system of parameters at the origin. Since we may write $\frac{\overline{\partial f}}{\partial x_{i}} \in G$ as $\frac{\partial f_{\rho}}{\partial x_{i}} \in \mathbf{C}\left[x_{1}, \ldots, x_{d}\right]=G$, Theorem 2 implies the relation

$$
e\left(\left(\frac{\partial f}{\partial x_{1}}, \ldots, \frac{\partial f}{\partial x_{d}}\right), \mathbf{C}\left\{x_{1}, \ldots, x_{d}\right\}\right)=\prod_{i=1}^{d}\left(\rho-w_{i}\right) \cdot \lim _{\lambda \rightarrow 1}(1-\lambda)^{d} P(G, \lambda) .
$$

\section{$\S 3$. Concluding Remarks}

Remark (3.1). (A similar result for a formal power series where the ground field is more general.) In their proof for the semi-quasihomogeneous complex analytic functions, Lê Dũng Tráng, Ramanujam and Arnol'd used topological arguments in 1] and [4. However, our proof is different from those and is purely algebraic. Since Theorem 2 holds for a more general situation, we can show a similar result for a formal power series over an arbitrary infinite field.

Let $k$ be an infinite field and $f \in k\left[\left[x_{1}, \ldots, x_{d}\right]\right]$. We assume $\left(\frac{\partial f}{\partial x_{1}}, \ldots, \frac{\partial f}{\partial x_{d}}\right)$ is a system of parameters of $k\left[\left[x_{1}, \ldots, x_{d}\right]\right]$. Then we define the Milnor number $\mu(f)$ as

$$
\mu(f)=\operatorname{dim}_{k} k\left[\left[x_{1}, \ldots, x_{d}\right]\right] /\left(\frac{\partial f}{\partial x_{1}}, \ldots, \frac{\partial f}{\partial x_{d}}\right) .
$$

Let $w=\left(w_{1}, \ldots, w_{d}\right)$ be a weight on the coordinates on $\left(x_{1}, \ldots, x_{d}\right)$ by positive integers $w_{i}, i=1, \ldots, d$, and let $f=f_{\rho}+f_{\rho+1}+\cdots$ be the weighted Taylor expansion with respect to $w$ with $f_{\rho} \neq 0$, where $f_{k}$ is a quasihomogeneous polynomial of type $\left(w_{1}, \ldots, w_{d+1} ; k\right)$.

We can show the following theorem by the same arguments as in the proof of Theorem 1.

Theorem 3. Let $k$ be an infinite field, and let $f \in k\left[\left[x_{1}, \ldots, x_{d}\right]\right]$ be as above. Then:

(1) We have the following inequality:

$$
\mu(f) \geq\left(\frac{\rho}{w_{1}}-1\right) \cdots\left(\frac{\rho}{w_{d}}-1\right) .
$$

(2) The equality holds in the above if and only if $\left(\frac{\partial f_{\rho}}{\partial x_{1}}, \ldots, \frac{\partial f_{\rho}}{\partial x_{d}}\right)$ defines a system of parameters of $k\left[x_{1}, \ldots, x_{d}\right]$.

We will give the following simple computations.

Example (3.2). (1) Let $f \in \mathbf{C}\{x, y, z\}$ be a unimodal function as follows:

$$
f=x^{p}+y^{q}+z^{r}+x y z, \quad 2 \leq p \leq q \leq r, \text { with } \frac{1}{p}+\frac{1}{q}+\frac{1}{r} \leq 1 .
$$


One can compute $\mu(f)$ as $\mu(f)=p+q+r-1$ (cf. [3]). We define a weight $w$ on the coordinates $(x, y, z)$ as $w=(q r, p r, p q)$. Then the initial term contains $x y z$ and $\rho=q r+p r+p q$. Our lower bound $\Pi$ is written as $\Pi=\frac{(q+r)(r+p)(p+q)}{p q r}$. Here we have the relation $\mu(f)-\Pi=(p+q+r)\left(1-\frac{1}{p}-\frac{1}{q}-\frac{1}{r}\right) \geq 0$.

(2) Let $f \in \mathbf{C}\left\{x_{1}, \ldots, x_{d}\right\}$ define a hypersurface isolated singularity of multiplicity $e$. Assume $f$ is written as follows: $f=x_{1}^{e}+\sum_{i=1}^{e} g_{i}\left(x_{2}, \ldots, x_{d}\right) x_{1}^{e-i}$ with $g_{i} \in\left(x_{2}, \ldots, x_{d}\right)^{i}$ for $i=1, \ldots, e$. Let $r$ be the integer defined by $r=$ $\min _{1 \leq i \leq e}\left[\frac{\operatorname{ord} g_{i}}{i}\right](\geq 1)$. We define a weight $w$ on the coordinates as $w\left(x_{1}\right)=r$, and $w\left(x_{i}\right)=1$ for $i=2, \ldots, d$. Then the initial term $f$ has the degree $\rho=r e$. Theorem 1 implies the inequality $\mu(f) \geq(e-1) \cdot(r e-1)^{d-1}$.

We will close this note by giving the following comment by the second named author (M. Tomari).

(3.3) One may ask whether one can get another proof of our results by following the studies of Kouchinirenko [3]. As remarked in the introduction, Furuya [2] showed the inequality (1) of Theorem 1 using 10 pages of combinatorial arguments about polyhedra. This is elementary but long, so does not necessarily show substantial points of this problem (I feel). In what follows, I consider a future approach and difficulties for proving the characterization of semi-quasihomogenity (2) following these lines a little. Let us employ the notation used in this paper.

Suppose the equality $\mu(f)=\left(\frac{\rho}{w_{1}}-1\right) \cdots\left(\frac{\rho}{w_{d}}-1\right)$ holds. There are many examples in which the Newton boundary $\Gamma(f)$ touches the hyperplane defined by the weight condition $\left(w_{1}, \ldots, w_{d} ; \rho\right)$ and has several maximal-dimensional faces (e.g., $f=x y+z^{m}+x^{n}+y^{n}$ with $n$ sufficiently large with respect to $m$ ).

So even in this case, the Newton filtration is not so simple as in the case of weight filtration. If the nondegenerate conditions are satisfied, the Poincare series of the associated graded rings of the Newton filtration have a nice inductive property [3]. However, in general, the treatment of this is difficult.

For simplicity, I assume that $\Gamma(f)$ has the unique maximal-dimensional face, and that $f$ is convenient. In particular, the support of $f_{\rho}$ contains $x_{i}^{\rho / w_{i}}$ for $i=1, \ldots, d$. Hence $f$ is a $\mu$-constant deformation of some Brieskorn singularity. Then there would some geometric approach to prove that $f_{\rho}$ is an isolated singularity. Or more generally, for the case that $\left(w_{1}, \ldots, w_{d}, \rho\right)$ is a quasi-smooth weight, a similar approach would work.

I hope that the studies along the line given by [3] will become more ring theoretical and involve our results in the future.

\section{ACKNOWLEDGMENT}

We the authors both heartily thank all members of the Tuesday Seminar at Nihon University (2000.4 -) and Waseda University (- 2000.3), which was organized by Prof. Kei-ichi Watanabe, for many encouragements. Upon those occasions, we could discuss our results and make the results known in this form. 


\section{REFERENCES}

[1] Arnol'd, V. I.: Normal forms of functions in the neighborhood of degenerate critical points, Uspekhi Mat. Nauk XXIX (1974), 11-49 = Russian Math. Surveys 29:2 (1974), 10-50. MR 58:24324

[2] Furuya, M.: Lower bound of Milnor number, preprint. (An early version of this is found in AG/9901107v2.)

[3] Kouchnirenko, A. G.: Polyèdres de Newton et nombres de Milnor, Invent. Math., 32 (1976), 1-31. MR 54:7454

[4] Lê Dũng Tráng and Ramanujam, C. P.: The invariance of Milnor's number implies the invariance of the topological type, Amer. J. Math., 98-1 (1976), 67-78. MR 53:2939

[5] Matsumura, H.: Commutative ring theory, Cambridge Studies in Adv. Math. 8, Cambridge University Press, Cambridge, 1986. (Japanese version was published in 1980.) MR 88h:13001

[6] Milnor, J.: Singular points of complex hypersurfaces, Annals of Math. Studies 61, Princeton University Press, Princeton, NJ, 1968. MR 39:969

[7] Milnor, J. and Orlik, P.: Isolated singularities defined by weighted homogeneous polynomials, Topology 9 (1970), 385-393. MR 45:2757

[8] Rees, D.: a-transforms of local rings and a theorem on multiplicities of ideals, Proc. Cambridge Philos. Soc. 57 (1961), 8-17. MR 22:9521

[9] Tomari, M.: Multiplicity of filtered rings and simple K3 singularities of multiplicity two, Publ. Res. Inst. Math. Sci., Kyoto Univ., 38 (2002), 693-724. MR 2003e:14029

[10] Tomari, M. and Watanabe, K.-i.: Filtered rings, filtered blowing-ups and normal twodimensional singularities with "star-shaped" resolution, Publ. Res. Inst. Math. Sci., Kyoto Univ. 25 (1989), 681-740. MR 91a:14010

2-155, Makinohara, Matsudo-city, Chiba, 270-2267, Japan

E-mail address: HZI00611@nifty.ne.jp

Department of Mathematics, College of Humanities and Sciences, Nihon University, Setagaya, Tokyo, 156-0045, Japan

E-mail address: tomari@math.chs.nihon-u.ac.jp 